\title{
Spectral peaks in electric field at resonance frequencies for seismically excited motion of ions in the Earth's magnetic field
}

\author{
Mayu Kuriki ${ }^{1}$, Masaki Matsushima ${ }^{1}$, Yasuo Ogawa ${ }^{2}$, and Yoshimori Honkura ${ }^{1}$ \\ ${ }^{1}$ Department of Earth and Planetary Sciences, Tokyo Institute of Technology, Tokyo 152-8551, Japan \\ ${ }^{2}$ Volcanic Fluid Research Center, Tokyo Institute of Technology, Tokyo 152-8551, Japan
}

(Received September 17, 2010; Revised January 14, 2011; Accepted March 25, 2011; Online published September 7, 2011)

\begin{abstract}
Clear electric field variations have been observed during the passage of seismic wave. The most notable feature is circular polarization of electric field and it has been interpreted as reflecting circular motion of ions in groundwater under the Earth's magnetic field. We have called such a mechanism 'seismic dynamo effect' but more convincing evidence is required to support this mechanism. Here we show strong evidence in terms of transfer function relating the electric field to the ground velocity due to seismic wave. We estimate transfer functions for electric field and ground velocity records for five aftershocks after the occurrence of $M 6.9$ earthquake. Then some peaks in the transfer function are found at specific frequencies where the resonance of ion motion in groundwater with the Earth's magnetic field is expected. This result clearly supports the mechanism, which provides a method for in-situ estimation of ion contents in groundwater.
\end{abstract}

Key words: Electric field, seismic wave, Earth's magnetic field, resonance, ground velocity.

\section{Introduction}

There are many studies on physical or chemical mechanisms to interpret electric field variations associated with earthquakes. Even for electric field variations associated with the passage of seismic wave, there are many examples (e.g. Thompson and Gist, 1993; Butler et al., 1996; Mikhailov et al., 1997; Beamish, 1999; Garambois and Dietrich, 2001; Haines et al., 2007) as well as theoretical studies (e.g. Pride and Morgan, 1991; Gershenzon, 1992; Pride, 1994; Pride and Haartsen, 1996; Haartsen and Pride, 1997; Garambois and Dietrich, 2002; Huang, 2002; Ren et al., 2010a, b). Appearance of unambiguous electric field changes was mostly synchronized with the arrival of seismic wave (Yamada and Murakami, 1982; Honkura et al., 2000, 2004; Mogi et al., 2000; Nagao et al., 2000; Matsushima et al., 2002; Ujihara et al., 2004). Recently we proposed a new mechanism for electric field generation by seismic wave or more specifically by ground motion, based on some observational examples (Honkura et al., 2009). Our claim is unique in that the Earth's magnetic field plays an essential role in generation of electric field, and hence we named the underlying mechanism as 'seismic dynamo effect' in an analogy with the dynamo theory for generation of planetary magnetism (e.g. Takahashi et al., 2005).

One peculiar observational feature is circular polarization of horizontal electric field variation and we succeeded in explaining this feature in terms of resonance-like motion of ion in groundwater under the Earth's magnetic field. In

Copyright (C) The Society of Geomagnetism and Earth, Planetary and Space Sciences (SGEPSS); The Seismological Society of Japan; The Volcanological Society of Japan; The Geodetic Society of Japan; The Japanese Society for Planetary Sciences; TERRAPUB.

doi:10.5047/eps.2011.03.010 particular, left-handed (counterclockwise) circular polarization, if seen towards the magnetic lines of force, is found to be associated with anions with negative electric charge. Similarly, right-handed (clockwise) circular polarization is associated with cations with positive charge. The magnitude of generated electric field is obviously proportional to the magnitude of ground velocity. It also depends on the number density of ions in groundwater. However, the most striking feature is the resonance-like behavior.

The resonance-like behavior would most effectively be examined by the frequency response of electric field to ground velocity. If we regard the two components of ground velocity, which are perpendicular to the Earth's magnetic field, as an input and the two components of the electric field as an output, we can examine frequency response function or transfer function. In magnetotelluric (MT) studies, such a transfer function is well known as impedance tensor, $Z_{i j}(i, j=1,2)$, with magnetic field variations as an input and electric field variations as an output. Here we use the same notation $Z_{i j}$, with the ground velocity, $\mathbf{v}_{0}$, as the input and the electric field, $\mathbf{E}$, as the output, as defined by

$$
\left(\begin{array}{c}
E_{x} \\
E_{y}
\end{array}\right)=\left(\begin{array}{ll}
Z_{11} & Z_{12} \\
Z_{21} & Z_{22}
\end{array}\right)\left(\begin{array}{c}
v_{0 x} \\
v_{0 y}
\end{array}\right)
$$

where the coordinate system, conventional in geomagnetism (northward horizontal direction $x$, eastward horizontal direction $y$ and downward direction $z$ ), is used. The dimension of $Z_{i j}$ is now $\mathrm{m}^{-2} \mathrm{~s} \mathrm{~V}$.

\section{Mechanism of Electric Field Generation}

Here we briefly review our theory for electric field generation (Honkura et al., 2009). The basic equation is given for aggregation of ion of mass $m$ and electric charge $q$ as

$$
d \mathbf{v} / d t+\mathbf{v} / \tau=q \mathbf{E} / m+\mathbf{v} \times \boldsymbol{\Omega}+\mathbf{v}_{0} \times \boldsymbol{\Omega},
$$


where $\tau$ is the relaxation time, and $\boldsymbol{\Omega}=m^{-1} q \mathbf{B}_{0}$ the cyclotron frequency in the Earth's magnetic field, $\mathbf{B}_{0}$, at the observation site. The drift velocity of the ion, $\mathbf{v}$, gives rise to the electric current, $\mathbf{j}=q \mathbf{v}$, in the ground moving with the velocity, $\mathbf{v}_{0}$, which is regarded as the forced velocity field exerted by seismic wave. We consider a periodic time variation expressed as $e^{-i \omega t}$, where $\omega$ is the angular frequency. Then the electric current, $\mathbf{j}$, due to electric polarization, $\mathbf{P}$, can be expressed as $\mathbf{j}=-i \omega \mathbf{P}$. Assuming that such $\mathbf{P}$ is uniformly distributed in a pore, we obtain approximate expression of the electric field, $\mathbf{E}$, in the pore as

$$
\mathbf{E} \sim-i N q \varepsilon^{-1} \omega^{-1} \mathbf{v},
$$

where $N$ is the number density of ions and $\varepsilon$ is the permittivity of groundwater.

In our model, ions motion is excited only on the plane perpendicular to the direction of $\mathbf{B}_{0}$, unless additional conditions such as the boundary conditions are imposed. Hence we consider the two components on the plane and rotate the coordinate system, $(x, y, z)$, by $90^{\circ}-I$ counterclockwise around the $y$-axis, where $I$ is the inclination of the Earth's magnetic field (about $50^{\circ}$ at the observation sites in this letter). Hereafter we denote the rotated system as $X$ and $Y$ (same as $y$ ) with new $Z$ corresponding to the direction of $\mathbf{B}_{0}$.

The $X$ and $Y$ components of an approximate solution of the above Eqs. (2) and (3) are then given as

$$
\begin{aligned}
& v_{X}=-\left(1-h_{\omega}^{2}\right)^{-1} \alpha^{-1}\left(1+\gamma^{2}\right)^{-1}(\gamma-i)\left(v_{0 X}+i h_{\omega} v_{0 Y}\right) \\
& v_{Y}=-\left(1-h_{\omega}^{2}\right)^{-1} \alpha^{-1}\left(1+\gamma^{2}\right)^{-1}(\gamma-i)\left(v_{0 Y}-i h_{\omega} v_{0 X}\right)
\end{aligned}
$$

where $h_{\omega}=\omega \Omega^{-1}, \alpha=N q\left(\varepsilon \omega B_{0}\right)^{-1}$ and $\gamma=(\alpha \tau \Omega)^{-1}$. The observed $x$ and $y$ components of electric field at the Earth's surface of the observation site are then derived as $-i \varepsilon_{\mathrm{b}}^{-1} \varepsilon \xi \alpha B_{0} v_{X} \sin I e^{-i \omega t}$ and $-i \varepsilon_{\mathrm{b}}^{-1} \varepsilon \xi \alpha B_{0} v_{Y} e^{-i \omega t}$, respectively, where $\varepsilon_{\mathrm{b}}$ is the permittivity of the matrix and $\xi$ the porosity. It is concluded, therefore, that the two components of electric field to be observed at the Earth's surface should be linearly related to $v_{0 X}$ and $v_{0 Y}$, respectively, and the concept of transfer function is applicable as in MT. Then from (4) and (5), resonance will be expected in transfer function for the observed electric field and the ground velocity, if $h_{\omega} \approx 1$, that is, if the frequency of ground motion is close to the cyclotron frequency for a certain kind of ion.

\section{Modification of Transfer Function}

We in fact found some peaks in transfer function estimates, as expected from resonance-like behavior, but the resonance frequencies for a few kinds of ion in groundwater turned out to systematically shift towards lower frequencies, as will be shown later. There must be a factor to be further considered in our model formulation. So far we have not considered any boundary conditions which may arise in the real Earth. The most likely condition is that the vertical component of electric field should vanish at the Earth's surface because the Earth is conducting. This condition implies that no vertical electric field should arise at the Earth's surface, or the vertical motion of ions must be prohibited near the Earth's surface. In view of this argument, we replace $B_{0}$ with $B_{0} \sin I\left(=B_{0 z}\right)$ and $v_{0 X}$ with $v_{0 X} \sin I$ as the $x$ component of ground velocity.

Summarizing the formulation with consideration of boundary condition, we can obtain the estimates of $Z_{i j}$ (1, 2 corresponding to $x, y$, respectively) with the $v_{0 X} \sin I$ and $v_{0 Y}$ as the $x$ and $y$ components of input, respectively, as

$$
\begin{aligned}
E_{x}= & -i \varepsilon_{\mathrm{b}}^{-1} \varepsilon \xi B_{0 z}\left(1-\hat{h}_{\omega}^{2}\right)^{-1}\left(1+\hat{\gamma}^{2}\right)^{-1} \\
& \cdot(\hat{\gamma}-i)\left(v_{0 x}+i \hat{h}_{\omega} v_{0 y}\right), \\
E_{y}= & -i \varepsilon_{\mathrm{b}}^{-1} \varepsilon \xi B_{0 z}\left(1-\hat{h}_{\omega}^{2}\right)^{-1}\left(1+\hat{\gamma}^{2}\right)^{-1} \\
& \cdot(\hat{\gamma}-i)\left(v_{0 y}-i \hat{h}_{\omega} v_{0 x}\right),
\end{aligned}
$$

and hence

$$
\begin{aligned}
& \left|Z_{11}\right|=\left|Z_{22}\right|=\lambda\left|\left(1-\hat{h}_{\omega}^{2}\right)^{-1}\right|, \\
& \left|Z_{12}\right|=\left|Z_{21}\right|=\lambda\left|\hat{h}_{\omega}\left(1-\hat{h}_{\omega}^{2}\right)^{-1}\right|,
\end{aligned}
$$

where $\hat{\Omega}=m^{-1} q B_{0 z}, \hat{h}_{\omega}=\omega \hat{\Omega}^{-1}, \hat{\alpha}=N q\left(\varepsilon \omega B_{0 z}\right)^{-1}$, $\hat{\gamma}=(\hat{\alpha} \tau \hat{\Omega})^{-1}$ and $\lambda=\varepsilon_{\mathrm{b}}^{-1} \varepsilon \xi B_{0 z}\left(1+\hat{\gamma}^{2}\right)^{-1 / 2} \cdot\left|Z_{i j}\right| \mathrm{de}-$ pends on various parameter values and its frequency dependence is not simple. However, if we focus our attention to a rather narrow frequency range near the resonance frequency, $\lambda$ turns out to decrease only slowly with $\hat{\gamma}$ which is proportional to $\omega$, at least as compared with more drastic resonance behavior, and hence $\left|Z_{11}\right|$ and $\left|Z_{22}\right|$ are regarded as approximately proportional to $\left|\hat{h}_{\omega}^{-1}\left(1-\hat{h}_{\omega}^{2}\right)^{-1}\right|$, and $\left|Z_{12}\right|$ and $\left|Z_{21}\right|$ to $\left|\left(1-\hat{h}_{\omega}^{2}\right)^{-1}\right|$.

\section{Results of Analyses}

To record temporal variations of the ground velocity and the electric field simultaneously for aftershocks of the M 6.9 mainshock which occurred on March 25, 2007 in central Japan, we installed a short-period seismometer of velocity type and two pairs of $\mathrm{Pb}-\mathrm{PbCl}_{2}$ electrodes. The separation of electrodes was 25 meters in both the NS and EW directions. The sampling frequency was $200 \mathrm{~Hz}$.

A clear example of electric field variations during the passage of seismic wave was obtained for an aftershock (M 4.3). The circular nature of electric field polarization has led us to a new physical model of electric field generation as briefly explained in the preceding sections (Honkura et al., 2009). In addition to this aftershock, smaller magnitude aftershocks turned out to provide useful data to further examine the mechanism. Figure 1(a) shows the hypocenters of five aftershocks including the one $(M 4.3)$ for which detailed analyses were made in Honkura et al. (2009). Figures 2(a) and 2(b) show seismic and electric field records for the $M 3.9$ aftershock which are used for transfer function analysis as introduced above. The transfer function is a statistical estimate and hence stacking is inevitable for reliable estimation. So we made the same analysis for all the aftershocks shown in Fig. 1(a).

The absolute values of stacked transfer function estimates are shown in Fig. 2(c). It is clear that the pair of $\left|Z_{11}\right|$ 

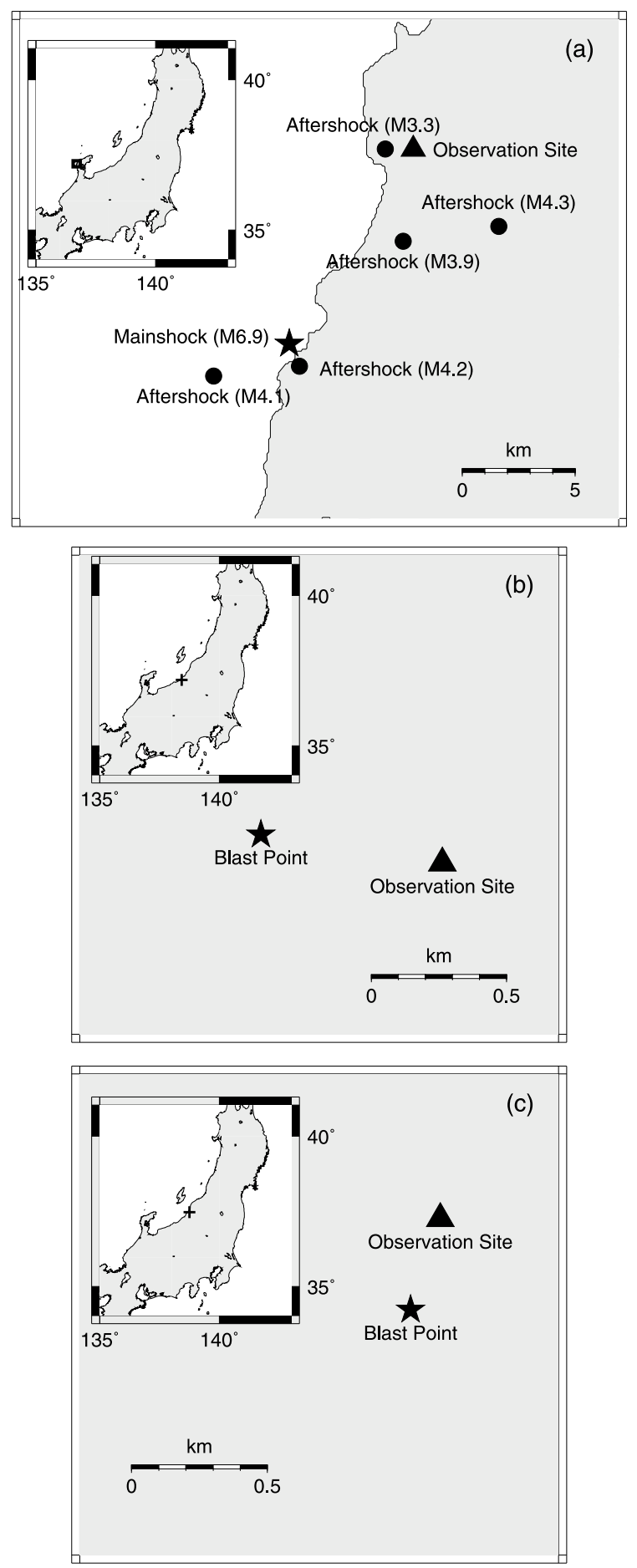

Fig. 1. (a) Location of the observation point and epicenters of the mainshock ( $M$ 6.9), which occurred on March 25, 2007 near the west coast of the Noto Peninsula, central Japan, and five aftershocks ( $M 4.3, M 4.2$, $M$ 4.1, $M$ 3.9, $M$ 3.3), for transfer function analysis shown in Fig. 2. (b) Location of the observation site and the blast point for transfer function analysis shown in Fig. 3. (c) Location of the observation site and the blast point for transfer function analysis shown in Fig. 4.

and $\left|Z_{21}\right|$ is much larger than the pair of $\left|Z_{12}\right|$ and $\left|Z_{22}\right|$ for some reason and hence the former is better determined. We can see clear three peaks in $\left|Z_{11}\right|$ and $\left|Z_{21}\right|$ near the resonance frequencies for $\mathrm{HCO}_{3}^{-}, \mathrm{Cl}^{-}$and $\mathrm{Na}^{+}$. It should be noted here that the resonance frequencies are shifted to lower frequencies, corresponding to the vertical component
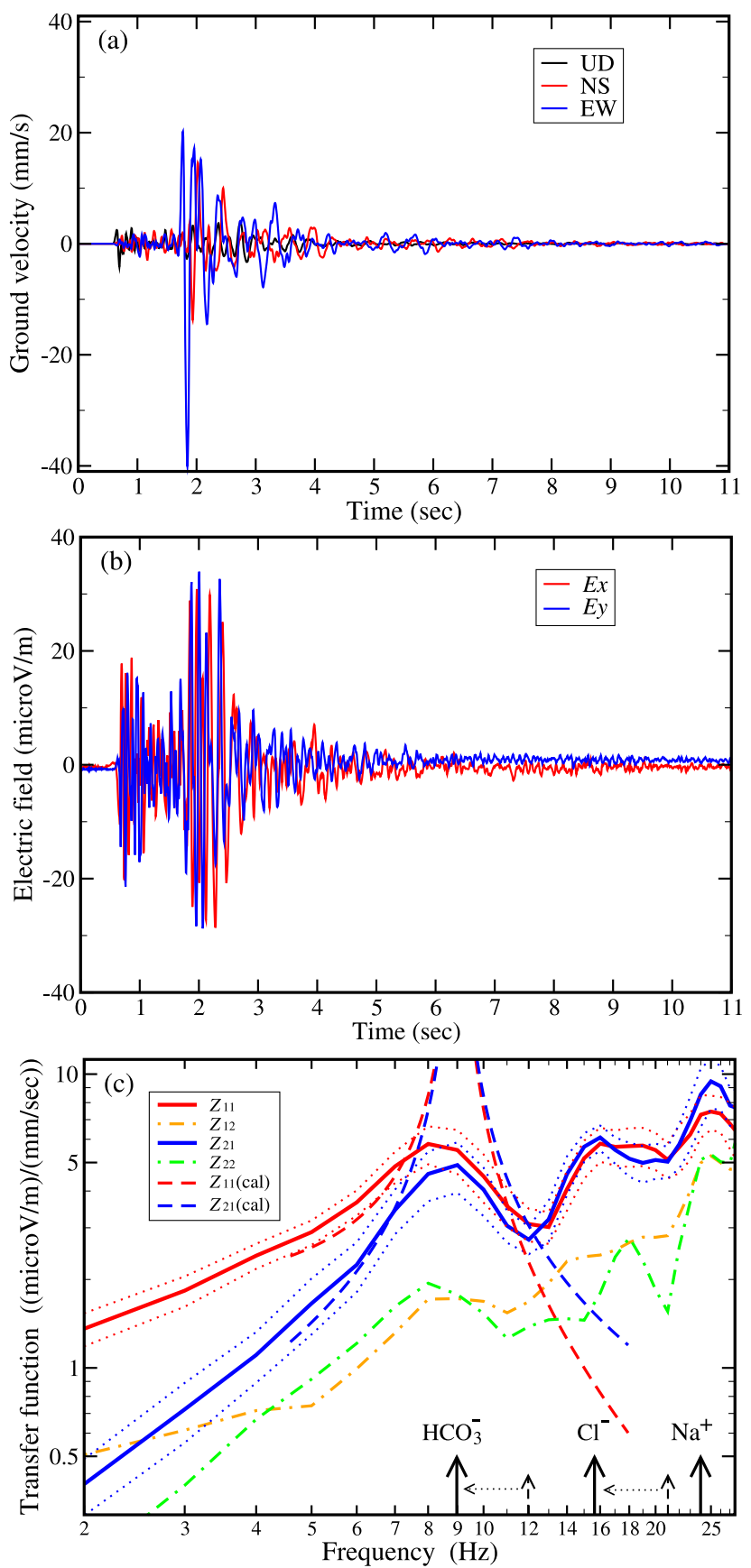

Fig. 2. (a) Ground velocity record of the $M 3.9$ aftershock and (b) electric field record, corresponding to the ground velocity. $E_{x}$ and $E_{y}$ denote the northward and the eastward components of electric field, respectively. (c) Absolute values of transfer function $Z_{i j}(i, j=1,2)$ derived from the observed ground velocity as the input and the observed electric field as the output. Dotted lines for $Z_{11}$ and $Z_{21}$ are standard errors. Dashed lines denote theoretical estimates for the resonance frequency of $9 \mathrm{~Hz}$, corresponding to $\mathrm{HCO}_{3}^{-}$.

of the Earth's magnetic field. The theoretical resonance responses, with the $\lambda$ value adjusted to data, for the resonance frequency of $9 \mathrm{~Hz}$ for $\mathrm{HCO}_{3}^{-}$are also shown for $\left|Z_{11}\right|$ and $\left|Z_{21}\right|$. The theoretical response is steep at the resonance frequency whereas the observed response is gradual. This is due partly to the smoothing operation, for example band averaging, inherent in spectral analysis and also partly to the fact that the ground velocity never keeps an exact resonance-frequency variation for a sufficiently long time 

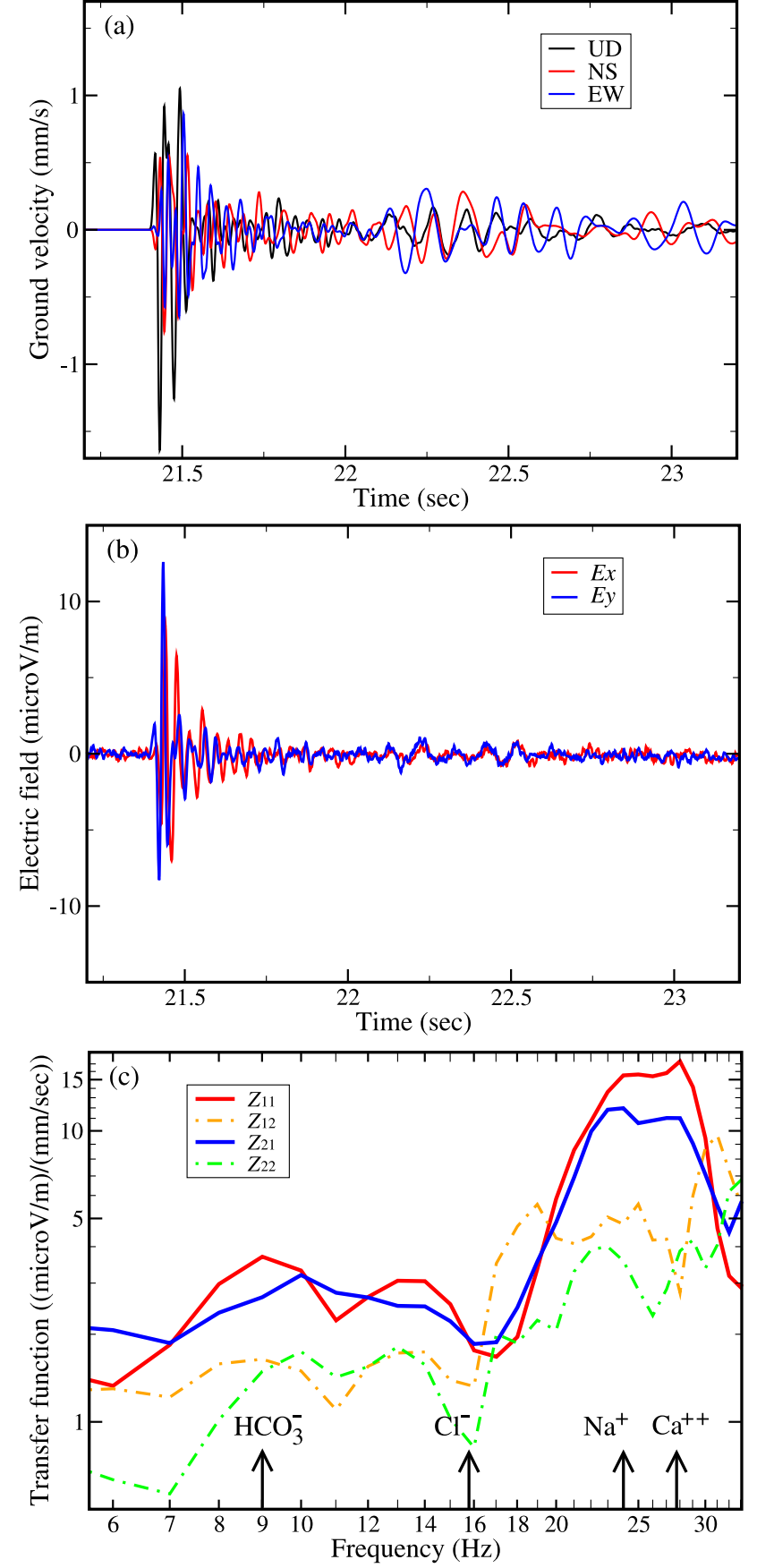

Fig. 3. (a) Ground velocity record of an artificial earthquake during a blasting experiment and (b) electric field record, corresponding to the ground velocity. $E_{x}$ and $E_{y}$ denote the northward and the eastward components of electric field, respectively. (c) Absolute values of transfer function derived from the observed ground velocity and the observed electric field.

so that the steady resonance state, as presumed in theoretical treatment, is never realized.

In the case of aftershocks shown above, the ground velocity is drastically reduced at frequencies higher than $30 \mathrm{~Hz}$ or so. In this respect, we already found that higher frequency components are available for the electric field and the ground velocity for artificial earthquakes generated by blasting (Honkura et al., 2009). Recently we made some observations in occasions of blasting and hence we also tried to estimate transfer functions for these records. It
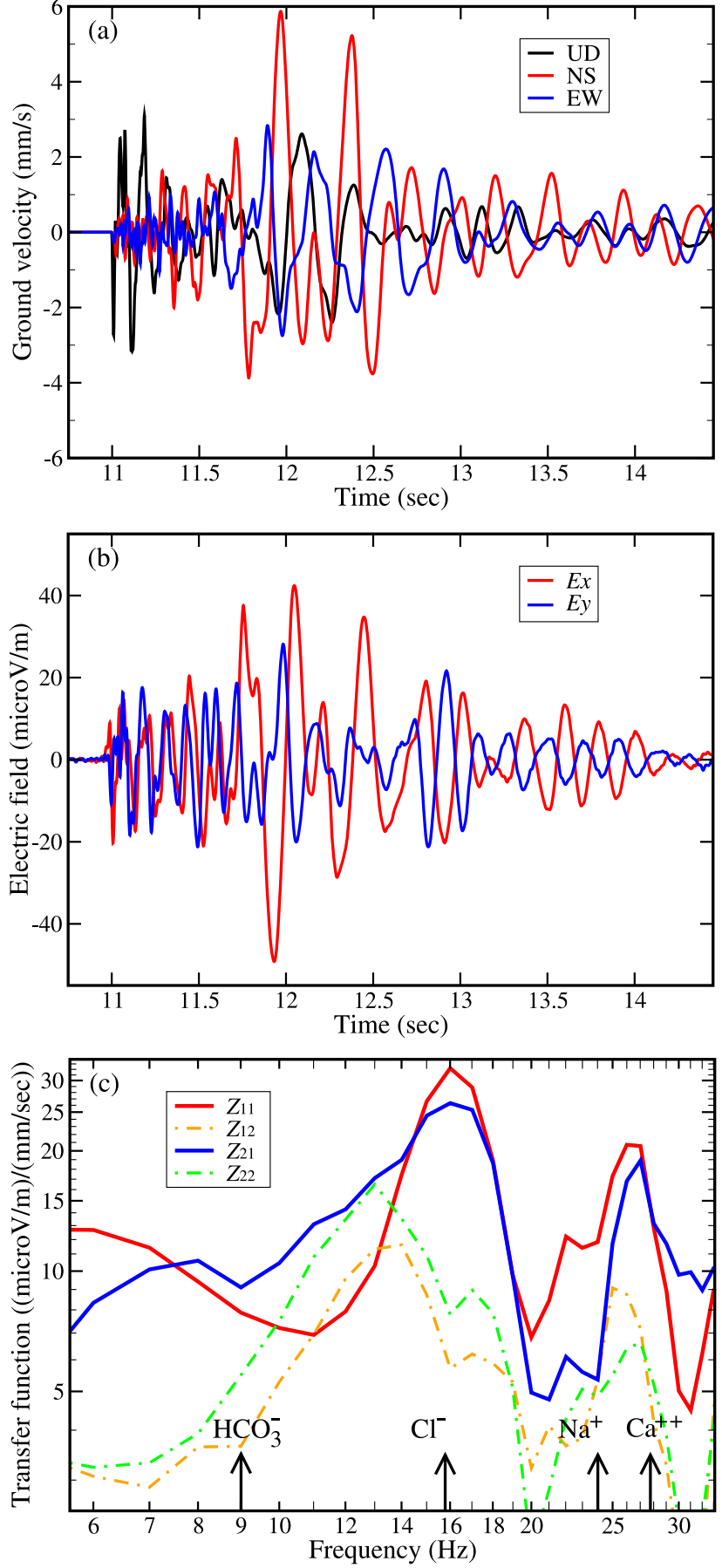

Fig. 4. (a) Ground velocity record of another artificial earthquake during another blasting experiment at another site and (b) electric field record, corresponding to the ground velocity. $E_{x}$ and $E_{y}$ denote the northward and the eastward components of electric field, respectively. (c) Absolute values of transfer function derived from the observed ground velocity and the observed electric field.

should be noted, however, that stacking essential for statistical properties is not possible in these cases and hence accuracy of transfer function estimates is worse than the case of aftershocks. Also, a higher sampling rate is required for transfer function estimates at higher frequencies. Recently, we introduced a data logger with $1 \mathrm{kHz}$ sampling, which turned out to be sufficient for frequencies lower than $40 \mathrm{~Hz}$, for example.

Figures 3(a) and 3(b) show electric field and ground ve- 
locity records used for transfer function estimates for the observation site and the blast point shown in Fig. 1(b). Higher frequency components seem to be dominant in the electric field record. In fact, transfer function estimates given in Fig. 3(c) seem to show a dominant peak near $25 \mathrm{~Hz}$ in $\left|Z_{11}\right|$ and $\left|Z_{21}\right|$ corresponding to the resonance frequency for $\mathrm{Na}^{+}$and/or $\mathrm{Ca}^{2+}$, even with rather poor transfer function estimates due to the lack of stacking in mind. Figure 4 shows another example of transfer function estimates for the observation site and the blast point shown in Fig. 1(c). In this case, two peaks are apparent in $\left|Z_{11}\right|$ and $\left|Z_{21}\right|$; one corresponding to the resonance frequency for $\mathrm{Cl}^{-}$, and the other probably to $\mathrm{Na}^{+}$.

\section{Discussion and Concluding Remarks}

In all the cases examined above, $\left|Z_{11}\right|$ and $\left|Z_{21}\right|$ turned out to be dominant compared with $\left|Z_{12}\right|$ and $\left|Z_{22}\right|$. In the theory proposed in Honkura et al. (2009), such anisotropy does not appear and hence there should be another factor to be considered in the theory. However, the present discussion on resonance-like response is little to do with this problem of anisotropy.

One may claim that the transfer function estimates should be clearer if the proposed mechanism is really in operation in groundwater. We admit such a claim but at the same time we point out one difficulty in the transfer function analysis which relates the two components of outputs (electric field) with the two components of inputs (ground velocity). One assumption is implicit in this treatment; two components of inputs are independent of each other. In reality, three components of ground velocity are somewhat correlated with each other and hence the $x$ and $y$ components are also correlated with each other to some extent. This problem would be overcome by stacking of many estimates, because correlations may change from one record to another. In fact, in the case of aftershocks, even stacking of five examples appreciably improved the quality of transfer function estimates. Such improvement due to stacking is in fact well known in magnetotellurics (MT).

We may now conclude that the appearance of peaks in the transfer function at the resonance frequencies, for $\mathrm{HCO}_{3}^{-}$, $\mathrm{Cl}^{-}$and $\mathrm{Na}^{+}$contained in groundwater, confirms the basic mechanism of electric field generation in association with the passage of seismic wave. Peak values of transfer function are indicative of relative amounts of respective ions at the site. In this sense, the mechanism is expected to provide a sort of in-situ ion analysis for groundwater.

Acknowledgments. We thank Professor Hiroshi Sato of Earthquake Research Institute, University of Tokyo, for providing us with the opportunity of our work during the blasting experiments. We also thank Japan Petroleum Exploration Co., Ltd. for providing us with an opportunity for our work during its seismic exploration. We are grateful to Dr. K. Yamazaki and an anonymous reviewer for their useful comments. This study was supported by the Japan Society for Promotion of Science (JSPS) under grant-inaid for scientific research No. 21340126.

\section{References}

Beamish, D., Characteristics of near-surface electrokinetic coupling, Geophys. J. Int., 137, 231-242, 1999.
Butler, K. E., R. D., Russell, A. W. Kepic, and M. Maxwell, Measurement of the seismoelectric response from a shallow boundary, Geophys., 61 , 1769-1778, 1996.

Garambois, S. and M. Dietrich, Seismoelectric wave conversion in porous media: field measurements and transfer function analysis, Geophys., 66, 1417-1430, 2001.

Garambois, S. and M. Dietrich, Full waveform numerical simulations of seismoelectromagnetic wave conversions in fluid-saturate stratified porous media, J. Geophys. Res., 107, doi:10.1029/2001JB000316, 2002.

Gershenzon, N. I., Seismoelectromagnetic field of an electrokinetic nature, Izvestiya Earth Phys., 28, 589-596, 1992.

Haartsen, M. W. and S. R. Pride, Electroseismic waves from point sources in layered media, J. Geophys. Res., 102, 24745-24769, 1997.

Haines, S. S., S. R. Pride, S. L. Klemperer, and B. Biondi, Seismoelectric imaging of shallow targets, Geophys., 72, G9-G20, 2007.

Honkura, Y., A. M. Işikara, N. Oshiman, A. Ito, B. Üçer, Ş. Bariş, M. K. Tunçer, M. Matsushima, R. Pektaş, C. Çelik, S. B. Tank, F. Takahashi, M. Nakanishi, R. Yoshimura, Y. Ikeda, and T. Komut, Preliminary results of multidisciplinary observations before, during and after the Kocaeli (Izmit) earthquake in the western part of the North Anatolian Fault Zone, Earth Planets Space, 52, 293-298, 2000.

Honkura, Y., H. Satoh, and N. Ujihara, Seismic dynamo effects associated with the M7.0 earthquake of 26 May 2003 off Miyagi Prefecture and the M6.2 earthquake in northern Miyagi Prefecture, NE Japan, Earth Planets Space, 56, 109-114, 2004.

Honkura, Y., Y. Ogawa, M. Matsushima, S. Nagaoka, N. Ujihara, and T. Yamawaki, A model for observed circular polarized electric fields coincident with the passage of large seismic waves, J. Geophys. Res., 114, B10103, doi:10.1029/2008JB006117, 2009.

Huang, Q., One possible generation mechanism of co-seismic electric signals, Proc. Japan Acad., 78, 173-178, 2002.

Matsushima, M., Y. Honkura, N. Oshiman, Ş. Bariş, M. K. Tunçer, S. B. Tank, C. Çelik, F. Takahashi, M. Nakanishi, R. Yoshimura, R. Pektaş, T. Komut, E. Tolak, A. Ito, Y. Iio, and A. M. Işikara, Seismoelecromagnetic effect associated with the Izmit earthquake and its aftershocks, Bull. Seismol. Soc. Am., 92, 350-360, 2002.

Mikhailov, O. V., M. W. Haartsen, and M. N. Toksöz, Electroseismic investigation of the shallow subsurface: field measurements and numerical modeling, Geophys., 62, 97-105, 1997.

Mogi, T., Y. Tanaka, D. S. Widarto, E. M. Arsadi, N. T. Puspito, T. Nagao, W. Kanda, and S. Uyeda, Geoelectric potential difference monitoring in southern Sumatra, Indonesia-Co-seismic change-, Earth Planets Space, 52, 245-252, 2000.

Nagao, T., Y. Orihara, T. Yamaguchi, I. Takahashi, K. Hattori, Y. Noda, K. Sayanagi, and S. Uyeda, Co-seismic geoelectric potential changes observed in Japan, Geophys. Res. Lett., 27, 1535-1538, 2000.

Pride, S., Governing equation for the coupled electromagnetics and acoustics of porous media, Phys. Rev., B50, 15678-15696, 1994.

Pride, S. R. and M. W. Haartsen, Electroseismic wave properties, J. Acoust. Soc. Am., 100, 1301-1315, 1996.

Pride, S. R. and F. D. Morgan, Electrokinetic dissipation induced by seismic waves, Geophys., 56, 914-925, 1991.

Ren, H., Q. Huang, and X. Chen, A new numerical technique for simulating the coupled seismic and electromagnetic waves in layered porous media, Earthq. Sci., 23, 167-176, 2010a.

Ren, H., Q. Huang, and X. Chen, Analytical regularization of the highfrequency instability problem in numerical simulation of seismoelectric wave-fields in multi-layered porous media, Chinese J. Geophys., 53, 506-511, 2010b.

Takahashi, F., M. Matsushima, and Y. Honkura, Simulations of quasiTaylor state geomagnetic field including polarity reversals on the Earth Simulator, Science, 309, 459-461, 2005.

Thompson, A. H. and G. A. Gist, Geophysical applications of electrokinetic conversion, Leading Edge, 12, 1169-1173, 1993.

Ujihara, N., Y. Honkura, and Y. Ogawa, Electric and magnetic field variations arising from the seismic dynamo effect for aftershocks of the M7.0 earthquake of 26 May 2003 off Miyagi Prefecture, NE Japan, Earth Planets Space, 56, 115-124, 2004.

Yamada, I. and H. Murakami, Self-potential variations associated with quarry blasts, Zisin, 35, 393-400, 1982.

M. Kuriki, M. Matsushima (e-mail: mmatsush@geo.titech.ac.jp), Y. Ogawa, and Y. Honkura 